\title{
A METHOD OF GRID OPTIMIZATION FOR FINITE ELEMENT METHODS*
}

\author{
Alejandro R. DIAZ, Noboru KIKUCHI and John E. TAYLOR \\ Department of Mechanical Engineering and Applied Mechanics, University of Michigan, \\ Ann Arbor, MI 48109, U.S.A.
}

Received 4 February 1983

Revised manuscript received 15 April 1983

\begin{abstract}
A computationally useful criterion for grid optimization is derived, based on a measure of the interpolation error associated with the finite element model. The result is intended to be used to improve the quality of finite element solutions by changing the location of the nodes within a fixed number of degrees of freedom. Examples of the application of the criterion are provided.
\end{abstract}

\section{Introduction}

The finite element method has become an important tool of engineering analysis and design. Much of the popularity of the method is due to the freedom that it allows in the construction of the discretized model. It has long been realized, however, that this freedom must be carefully exercised since the quality of the finite element solution greatly depends on how the discretization is performed. Several methods have been proposed to improve the discretized model in an iterative manner. One such method is presented in this paper.

A natural way of improving the quality of finite element solutions is to increase the number of degrees of freedom. The process is normally performed after an initial solution is already available. Several schemes have been devised to introduce the new degrees of freedom in a selective manner in order to produce the greatest possible improvement of the previous solution. This calls for the definition of criteria to identify the regions of the domain where the finite element approximation is poorer. The new degrees of freedom are added in these regions by either increasing the order of polynomial approximation inside elements, the so-called $p$-method, or by subdivision of elements, or $h$-method. The process is continued until a specified accuracy is achieved.

The quality of the finite element solution may be improved also by optimizing the disposition of the nodes. Analysts often rely on their experience to construct grids that make an efficient use of the available degrees of freedom. It is also possible to improve the quality of existing meshes iteratively using predefined guidelines for the redistribution of the nodes. This paper considers the development of such guidelines.

*The authors are supported by NSF, CEE-8118158 in order to work the present subject, and express their appreciation to NSF.

0045-7825/83/\$3.00 (C) 1983, Elsevier Science Publishers B.V. (North-Holland) 


\subsection{Summary of existing grid optimization methods}

An example of the implementation of an $h$-version of the finite element method is presented in [1-4]. In these works, new degrees of freedom are introduced by selectively subdividing elements in regions where the finite element approximation is less accurate. These regions are identified by means of element error 'indicators' constructed from the finite element solution. The 'indicators' are related to the size of each element and to local residuals that arise from the force unbalance resulting from the application of the original differential operator to the finite element solution. An enriched mesh is obtained after subdividing, in a nested fashion, all elements with high error 'indicators'. For one-dimensional grids it is shown that near-optimality is achieved when the error 'indicators' are constant for all elements. This result is also applied to two-dimensional problems. Error 'indicators' are also used to estimate the magnitude of the finite element error $e$. An error 'estimator' $\varepsilon$ is introduced and the authors discuss the conditions under which $\|e\| \approx \varepsilon$, where the $L^{p}$-stress-energy norm is used. For many one-dimensional problems the 'estimator' is asymptotically exact in the sense that $\varepsilon /\|e\| \rightarrow 1$ as the representative size of the element approaches zero. Other error indicators, based on interpolation crror bounds similar to those described later in this paper, are discussed in [5]. In this case the author proposes a form of $h$-enrichment of the mesh that applies to triangular elements.

The selective enrichment of the mesh by the $p$-method is treated in $[4,6,7]$. Also explored are the advantages of using hierarchical interpolating functions within the context of both the $h$-version and the $p$-version of mesh enrichment. When hierarchical functions are used, the introduction of an additional degree of freedom has the effect of adding an extra term to the approximation of the solution, the usual analogy being the addition of terms in a Fourier series. Further computational efficiency is achieved since the 'stiffness' matrix corresponding to the initial mesh becomes a submatrix of the 'stiffness' matrix associated with the enriched mesh. Error 'indicators' and 'estimators' based on residuals are also used in these papers to identify the regions where new degrees of freedom should be added. Other references on hierarchical elements are found in $[8,9]$.

Much of the work in grid optimization has been based on the fact that the finite element solution yields an upper bound on the potential energy. Given a fixed number of elements and a specified order of local polynomial approximation, the best grid is defined as the one associated with the lowest upper bound on the potential energy. The problem may be stated as follows: find the location of the nodes $\boldsymbol{x}$ in order to

$$
\begin{array}{ll}
\text { minimize } & \Pi\left(x, u_{h}(x)\right), \\
\text { subject to } & \text { geometry constraints . }
\end{array}
$$

Here $I I$ represents the potential energy and $u_{h}(x)$ denotes the finite element solution corresponding to $\boldsymbol{x}$. Unfortunately, the complexity of the relation between $\boldsymbol{x}$ and $u_{h}$ makes the formal solution to this problem generally unattractive. Rather than attempt to find a true minimizer of $\Pi$, several authors have described guidelines that approximate in some sense the true optimality conditions and are at the same time easier to implement computationally.

The strain energy density (sed) of the approximate solution has been used in different forms for the development of guidelines based on the reduction of the potential energy. A rule that 
suggests elements should be disposed along lines of constant sed, or isoenergetics, was first proposed in [10]. The same suggestion is made in [11] after the authors examine several numerical examples in which the potential energy was minimized using a direct search technique. The authors also propose a scheme for the selection of the appropriate isoenergetic contours and for the placement of nodes on these lines. A similar method is studied in [12].

A study based on the direct minimization of the potential energy is presented in $[13,14]$. The author uses mathematical programming methods that do not require derivative information and combines them with a 'node distribution' scheme based on local energy distribution for improved convergence.

Another optimality criterion based on sed is presented in [15], where the authors study the effect that the 'gradual' introduction of an additional degree of freedom has on the potential energy. This effect is measured by the difference of strain energy densities in the enriched and the original meshes. This quantity is defined as the specific energy difference. The magnitude of the strain energy difference associated with the introduction of a degree of freedom within an element is estimated by taking the difference between the sed at any point and the sed at the centroid of the element. This measure provides the basis for the evaluation of the merit of a grid and mesh refinement is adopted in regions of high strain energy difference.

A procedure involving the use of interactive computer graphics for the construction of improved grids is presented in [16]. Here it is argued that the best finite element solution is the one that best approximates the total strain energy. This solution is associated with the best possible approximation to the integrand of the strain energy, the sed. In order to reduce the error involved in this approximation, the authors propose that nodes should be distributed in such a way that the difference of seds between selected neighboring nodes is made constant.

The criteria and procedures mentioned above constitute a small but significant sample of the different approaches to mesh optimization. The list is by no means complete and important contributions have not been included because of limitations in space. The reader is referred to $[12,17]$ for additional references.

\subsection{Present work}

The addition of degrees of freedom by the $h$-or $p$-methods is an attractive means to improve the finite element approximation and indeed this may be the recommended approach for some problems. After all, convergence to the exact solution is in all reasonable examples insured if the process of refinement is continued indefinitely. In some instances however the analyst may be interested only in improving an existing grid without the complications involved in subdividing elements or in using different interpolating functions in different elements. This is often the case, for instance, when finite elements are used as part of an iterative design optimization process. The modification of the grid may be incorporated as part of the design scheme with only moderate additional effort, especially if the criteria for grid optimization resemble the optimality criteria for the design.

The iterative improvement of a grid is equivalent to an optimal design remodelling problem based on optimality criteria. In such problems, the analyst is given an initial design on which a fixed amount of remodelling is performed and the task consists on applying the optimality criteria to modify the design in the most efficient way. In order for this approach to be feasible, the optimality criteria must appear in a form that allows easy implementation. The 
requirement that some quantity be constant over certain regions is an often encountered form of optimality criterion. Equally distributed stresses or constant strain energy density on the domain boundary are typical examples. Conditions of this form are also desirable in grid optimization. If $f_{K}$ is a quantity defined over element $K$, guidelines for grid optimization such as $f_{K}=$ constant for all elements $K$ can be implemented using already existing remodelling schemes. The parallel improvement of both grid and design would be simplificd as well. A simple version of such guideline is proposed in this paper.

Most rules for grid optimization are related to the minimization of a specific quantity, or objective function, selected as the measure of quality of the grid. The objective selected in this paper is the error associated with the interpolation of the true solution using functions from the finite element space. This is a reasonable choice since, for well posed problems, the interpolation error bounds the finite element error. This use of the interpolation error also permits the derivation of useful guidelines for grid remodelling. The problem is presented as an optimal design formulation. The objective function is constructed from existing bounds on interpolation error, available from interpolation theory. Element lengths and areas are selected as design variables, respectively in one- and two-dimensional problems. The necessary conditions for a minimum of this problem appear in a form unsuitable for computations but they can be successfully replaced by more manageable equations that become the desired guidelines. The effect of this approximation may be measured in terms of the representative size of the mesh and, in all cases studied, the proposed guidelines have produced good results.

\section{Finite element approximation}

The magnitude of the finite element error and of the interpolation error and its bound are measured here using the norms and seminorms associated with the Sobolev spaces $H^{m}(\Omega)$. These spaces are formed by functions whose generalized derivatives of order $m$ are bounded, in the mean square sense, over the domain $\Omega$. In two dimensions, the space $H^{m}(\Omega)$ equipped with the norm

$$
\|v\|_{m, \Omega}=\left\{\int_{\Omega} \sum_{\alpha_{1}+\alpha_{2} \leqslant m}\left[\frac{\partial^{\left(\alpha_{1}+\alpha_{2}\right)} v}{\partial x_{1}^{\alpha_{1}} \partial x_{2}^{\alpha_{2}}}\right]^{2} \mathrm{~d} x\right\}^{1 / 2}
$$

and seminorm

$$
|v|_{m, \Omega}=\left\{\int_{\Omega} \sum_{\alpha_{1}+\alpha_{2}=m}\left[\frac{\partial^{\left(\alpha_{1}+\alpha_{2}\right)} v}{\partial x_{1}^{\alpha_{1}} \partial x_{2}^{\alpha_{2}}}\right]^{2} \mathrm{~d} x\right\}^{1 / 2}
$$

where $\alpha_{1}$ and $\alpha_{2}$ are nonnegative integers. Similar expressions apply to one-dimensional domains by simply setting $\alpha_{2}$ to zero.

Let the original problem be a second-order, elliptic boundary value problem. Let $u$ be the exact solution of the associated variational problem

$$
a(u, v)=F(v) \text { for all } v \in V
$$

where $a(\cdot, \cdot)$ is a bilinear, bicontinuous, and $V$-elliptic form on $V \times V ; F(\cdot)$ is a bounded linear 
form on $V$; and $V$ is a subspace of Sobolev spaces that usually includes some of the boundary conditions. Let $u_{h}$ be the corresponding finite element approximation selected from a finite-dimensional space $X_{h}$. It is assumed that all the necessary conditions for the existence of $u$ and $u_{h}$ are satisfied. It is also assumed that the original domain is polygonal and therefore, it may be covered by straight sided elements. Certain simplifications apply also to the geometry of the elements. Only line, triangular and quadrilateral elements are considered. Using the definition of [18, Chapter 3], it is required that all elements be regular. This requirement is satisfied by all elements that are not 'collapsed', or equivalently, whose internal angles are bounded away from $0^{\circ}$ and $180^{\circ}$. Finally, secondary nodes, if present, are placed so that the corresponding side is subdivided into equal segments.

Assuming that the solution $u$ is smooth enough, it may be interpolated using functions from the finite-dimensional space $X_{h}$. Let $u_{I}$ be such a function. For all nodes $b_{j}, u_{I}$ satisfies

$$
u_{I}\left(b_{j}\right)=u\left(b_{j}\right), \quad j=1,2, \ldots, N,
$$

i.e., $u_{I}$ has the same value of $u$ at the nodes and it may be represented using the standard interpolating functions inside each element. The function $u_{I}$ is introduced because, for well posed problems, the error associated with the interpolation of $u$ by $u_{I}$ bounds the error of approximating $u$ by $u_{h}$. That is,

$$
\left\|u-u_{h}\right\| \leqslant C\left\|u-u_{I}\right\|
$$

for some positive constant $C$. The norm $\|\cdot\|$ in (3) above is the norm induced by $a(\cdot, \cdot)$ on $V$ and, for many significant problems, it is equivalent to a norm in $H^{1}(\Omega)$. This result has been used extensively to construct estimates of the rate of convergence of the finite element method. Bounds on the interpolation error are immediately available for that purpose. For the purposes of the grid optimization problem, these same bounds are used the basis for the construction of objective functions. The following result from interpolation theory provides an excellent bound on the error.

\section{Interpolation error theorem}

Let the space of finite element solutions be of degree $k \geqslant 1$, and let $u \in H^{k+1}(\Omega)$. Define $h_{K}$ as the diameter of element $K$. There exists a positive constant $C$ independent on $K$ such that, for all finite elements,

$$
\left\|u-u_{I}\right\|_{m, K} \leqslant C h_{K}^{k+1-m}|u|_{k+1, K}, \quad 0 \leqslant m \leqslant k .
$$

This result constitutes an excellent basis for the construction of guidelines for grid optimization. It relates the local interpolation error, and therefore the finite element error, to local functions of element sizes. Furthermore, a global measure of the error is available by the simple addition of these terms.

It is useful to introduce a function $B(u, h)$, with $\boldsymbol{h}=\left(h_{1}, h_{2}, \ldots, h_{M}\right)$, defined by 


$$
B=\left\{\sum_{K=1}^{M} h_{K}^{\beta}|u|_{k+1, K}^{2}\right\}^{1 / 2}
$$

where $\beta$ is a given parameter. In most problems the norm in (3) is equivalent to a norm in $H^{1}(\Omega)$, and, using (4) and the definition of $B$, it follows that

$$
\left\|u-u_{h}\right\|_{1, \Omega} \leqslant C B(u, \boldsymbol{h}), \quad \beta=2 k .
$$

A similar result in terms of element areas $A_{K}$ is also possible. For regular elements there exists a constant $\sigma$ independent on $K$, such that

$$
h_{K}^{2} \leqslant \sigma A_{K}, \quad K=1,2, \ldots, M .
$$

Using (4),

and

$$
\left\|u-u_{I}\right\|_{m, K} \leqslant \sigma C A^{1 / 2(k+1-m)}|u|_{k+1, K}
$$

$$
\left\|u-u_{h}\right\|_{1, \Omega} \leqslant \sigma C B(u, \boldsymbol{A}), \quad \beta=k
$$

where $A=\left(A_{1}, A_{2}, \ldots A_{M}\right)$. Clearly, some of the accuracy of the original bound is lost in this step.

\section{The grid optimization problem}

The function $B$ is the basis for the construction of the guidelines for grid optimization. For one-dimensional problems $B$ is expressed as a function of element lengths. For two-dimensional grids, element areas are used instead. The value of the parameter $\beta$ is varied accordingly. Stated in the form of a minimization problem, the grid optimization problem takes the following forms.

(1) One-dimensional grids. Find the vector of element lengths $\boldsymbol{h}=\left(h_{1}, h_{2}, \ldots, h_{M}\right)$ that

$$
\begin{array}{cl}
\text { minimizes } & B^{2}(u, \boldsymbol{h})-\sum_{K=1}^{M} h_{K}^{\beta}|u|_{k+1, K}^{2}, \\
\text { subject to } & \sum_{K=1}^{M} h_{K}=1 \\
& h_{K} \geqslant 0
\end{array}
$$

where $\beta=2 k$ and the domain has been normalized.

(2) Two-dimensional grids. Given a polygonal domain $\Omega$ of fixed boundary, find the vector of element areas $\boldsymbol{A}=\left(A_{1}, A_{2}, \ldots, A_{M}\right)$ that

$$
\text { minimizes } B^{2}(u, \boldsymbol{A})=\sum_{K=1}^{M} A_{K}^{\beta}|u|_{k+1, K}^{2},
$$




$$
\begin{array}{ll}
\text { subject to } & \sum_{K=1}^{M} A_{K}=1, \\
& A_{K} \geqslant 0 .
\end{array}
$$

A set of guidelines for the construction of approximations to the solution of problem (9) can be derived using a trick introduced by Babuska in [2, p. 441]. The trick is based on the use of a so-called grading function $p(t)$ to define the disposition of the nodes on the domain. This function $p(t):[0,1] \rightarrow[0,1]$ is continuous, with continuous first derivative, and strictly increasing in $(0,1)$. The set of nodes $\left\{0=x_{0}<x_{1}<\cdots<x_{M}=1\right\}$ is called a graded partition of the domain if it satisfies the conditions

where

$$
p\left(x_{K}\right)=K / M, \quad K=0,1,2, \ldots, M
$$

$$
p(0)=0, \quad p(1)=1 .
$$

With the aid of $p(t)$, it is possible to express the objective $B^{2}(u, h)$ in $(9 . a)$ as a functional with $p(t)$ as the dependent variable. This is done by first replacing the element lengths $h_{K}$ using

$$
\int_{X_{K-1}}^{X_{K}} p^{\prime}(t) \mathrm{d} t=1 / M=p^{\prime}\left(x_{K-1 / 2}\right) h_{K}\left(1+\mathrm{O}\left(h_{K}\right)\right),
$$

with $x_{K-1 / 2}=1 / 2\left(x_{K-1}+x_{K}\right)$, and then approximating the sum over all elements in (9.a) by

$$
B^{2}=\frac{1}{M^{\beta}} \int_{0}^{1} \frac{g(t)}{\left[p^{\prime}(t)\right]^{\beta}} \mathrm{d} t(1+\mathrm{O}(h))
$$

Here the symbol $g(t)$ is used to represent the argument in the integral of the seminorm $|u|_{k+1, \Omega}$. The approximation of $B^{2}$ suggests the use of the calculus of variations to obtain the function $p(t)$ that minimizes the integral. This optimum grading function satisfies

$$
p^{\prime}(t)=\frac{g(t)^{1 / \beta+1}}{\int_{0}^{1} g(t)^{1 / \beta+1} \mathrm{~d} t} .
$$

Using (11) and (13), the condition of optimality of $p$ is asymptotically equivalent to the set of equations

$$
f_{K} \equiv h_{K}^{\beta}|u|_{k+1, K}^{2}=\lambda, \quad K=1,2, \ldots, M
$$

where $\lambda$ is a constant, independent of $K$, and $\beta=2 k$. Furthermore, as a result of the introduction of the grading function, it is possible to show that if $h^{*}$ denotes the true optimum and $h$ is the approximation from (14),

$$
B^{2}(u, h)=B^{2}\left(u, h^{*}\right)(1+O(h))
$$

where $h=\max _{K}\left\{h_{K}\right\}$. 
Guidelines (14) are quite naturally related to the local interpolation error. In view of (4), (14) indicate that the grid $\boldsymbol{h}$ makes the element interpolation error roughly the same throughout the domain. The derivation of a similar result for application to two-dimensional problems is not as simple. The guidelines for grid optimization in two dimensions proposed here is simply an extension of the one-dimensional result, namely

$$
f_{K} \equiv A_{K}^{\mathcal{B}}|u|_{k+1, K}^{2}=\lambda, \quad K=1,2, \ldots, M
$$

where $\beta=k$. This extension is in part motivated by the observation that the true optimality conditions of problems (9) and (10) also have a similar form. For completeness, these equations are presented below and their derivation is outlined in Appendix A. Using the symbol $m_{K}$ to represent the $\mathbb{R}^{n}$-measure of element $K$ (length in $\mathbb{R}^{1}$, area in $\mathbb{R}^{2}$ ), the true necessary conditions for optimality of (9) or (10) indicate that for all elements $K$ and neighbors $N$ such that $K \cap N \neq \emptyset$,

$$
g_{K N} m_{K}^{\beta}+\beta m_{K}^{\beta-1}|u|_{k+1, K}^{2}=g_{K N} m_{N}^{\beta}+\beta m_{N}^{\beta-1}|u|_{k+1, N}^{2} .
$$

Here $g_{K N}$ is the average value of $g(t)$ on the side or node shared by $K$ and $N$ (see Appendix A). Given that the same equation (17) describes optimality in either $\mathbb{R}^{1}$ or $\mathbb{R}^{2}$, it is reasonable to simplify the problem in $\mathbb{R}^{2}$ by extending the one-dimensional guideline (14) into two dimensions via (16).

Equations (14) and (16) are of the right form, namely $f_{K}=$ constant, but because of the presence of the exact solution $u$, they are still not usable for computations. At this point it is necessary to find a suitable replacement for $|u|_{k+1, k}$. Several alternatives are possible and an easy to compute substitute is proposed here. Let the solution $u$ be replaced in $|u|_{k+1, K}$ by a known function $\hat{u}$. The function $\hat{u}$ should be available from the finite element solution and should be such that the ratio $|u-\hat{u}|_{k+1, \Omega} /|u|_{k+1, n}$ is small, of order $h^{\alpha}$ for some positive number $\alpha$. Using $\hat{u}$, the suggested guidelines take the final form

$$
\begin{array}{ll}
\text { (1-D) } \quad h_{K}^{\beta}|\hat{u}|_{k+1, K}^{2}=\hat{\lambda}, & \beta=2 k, \\
\text { (2-D) } \quad A_{K}^{\beta}|\hat{u}|_{k+1, K}^{2}=\hat{\lambda}, \quad \beta=k .
\end{array}
$$

The following simple construction of $\hat{u}$ for use in the seminorm $|\hat{u}|_{k+1, K}$ has been used with reasonable success.

(1-D): At each of the element end points, the average value of $\partial^{k} u_{h} / \partial x^{k}$ across the element boundary is evaluated. Using these two values and the magnitude of $\partial^{k} u_{h} / \partial x^{k}$ at the center of the element, a piecewise linear function is defined inside each $K$. This function corresponds to $\partial^{k} \hat{u} / \partial x^{k}$.

(2-D): For each nonnegative pair $\left(\alpha_{1}, \alpha_{2}\right)$ such that $\alpha_{1}+\alpha_{2}=k$, the average value of $\partial^{\alpha_{1}+\alpha_{2}} u_{k} / \partial x_{1}^{\alpha_{1}} \partial x_{2}^{\alpha_{2}}$ across the element boundary is evaluated at each element midside. These point values are interpolated inside the element and the result is defined as $\partial^{\alpha_{1}+\alpha_{2}} \hat{u} / \partial x_{1}^{\alpha_{1}} \partial x_{2}^{\alpha_{2}}$.

This construction of $\hat{u}$ demands a minimum of additional computational effort and, under reasonable assumptions on the regularity of $u$, it corresponds to ratio $\left.|u-\hat{u}|_{k+1, \Omega}|| u\right|_{k+1, \Omega}$ of order $h^{1 / 2}$, although higher values have been observed $(\alpha=1)$. The proposed guidelines have 
been used in problems with singularities, where the solution is no longer in $H^{k+1}(\Omega)$ and, even in these cases, remarkable improvements in the quality of the approximation $u_{h}$ are achieved. For linear interpolation models $(k=1)$, the seminorm $|\hat{u}|_{k+1, K}$ is related to stress jumps across element boundaries in problems in mechanics. The conditions (18) and (19) correspond then to making some weighted average of these jumps constant throughout the domain. Other definitions of $\hat{u}$ are possible and the one suggested here was selected for the simplicity of its computation.

\section{Computer implementation}

An iterative algorithm was developed to modify existing grids to satisfy the conditions

$$
f_{K}=A_{K}|\hat{u}|_{k+1, K}^{2}=\text { constant }
$$

discussed earlier. The method was conceived as a simple but secure way of obtaining improved grids without extensive manipulations. The procedure was selected, not for its computational efficiency in terms of CPU time, but because of the simplicity of its implementation. A similar scheme can be applied to the simpler one-dimensional problem, but it is not discussed here due to space limitations.

The modification of two-dimensional grids presents the difficulty that the movement of a node requires the knowledge of both the direction and the magnitude of the change. The motion of each node must be consistent with the boundary of the domain and with the connectivity of the mesh, and large enough to produce quick improvement. Extensive grid modification per iteration, however, demands somewhat complicated book-keeping schemes and it is not attempted here for the sake of simplicity.

The motion of a node is determined by the magnitude of the $f_{K}$ 's in elements surrounding the node. If the value of $f_{K}$ is larger than average, the nodes around $K$ should move so that $A_{K}$ is reduced. Since a node is usually shared by several elements, its local movement should be in the direction of the neighboring element with the larger $f_{K}$. The magnitude of the movement depends on the relative magnitude of the $f_{K}$ 's. This suggests the idea of assigning a 'mass-like' quantity to each element. The 'mass' is placed at the element's geometric center and 'attracts' the nodes surrounding it. At each $\nu$ iteration, this motion is described by

where

$$
x_{n}^{\nu+1}=\frac{\sum_{K \in\{N\}} \bar{X}_{K}^{\nu}\left(F_{K} / A_{K}\right)^{\nu}}{\sum_{K \in\{N\}}\left(F_{K} / A_{K}\right)^{\nu}}
$$

$x_{n}^{\nu+1}$ is the new location of the $n$th node;

$\{N\}$ is the set of all elements that contain $x_{n}$;

$\bar{X}_{K}^{\nu}$ is the location of the geometric center of $K$ in the $\nu$ th iteration.

For nodes on the boundary, the motion is simply restricted to be tangent to the boundary. This is the only precaution needed to insure that the shape of the domain remains unchanged.

The method has been successfully tested using quadrilateral elements, although no convergence proof is available. Improvement per iteration depends on the behavior of the 
solution $u$ and on the initial, given grid. However, in most cases studied, it has not been necessary to satisfy the conditions (20) exactly in order to achieve a substantial improvement. Most of the improvement is already available when the $f_{K}$ 's are simply of the same order of magnitude.

\section{Examples}

The following examples of improved grids were constructed by modifying an initial grid-typically uniform-to satisfy the guidelines for grid optimization given by (18) or (19). In most cases a substantial improvement of the finite element solution is available from grids that satisfy these equations only approximately. In these cases the modification of the grid is stopped before the guidelines are satisfied exactly.

EXAMPLE 6.1. The first example corresponds to the solution of the one-dimensional problem

$$
\begin{aligned}
& u \frac{\mathrm{d} T}{\mathrm{~d} x}=\frac{\mathrm{d}}{\mathrm{d} x}\left(k \frac{\mathrm{d} T}{\mathrm{~d} x}\right), \quad x \in(0,1) \\
& T(0)=0, \quad T(1)=1
\end{aligned}
$$

with $u=1$, constant in $(0,1)$, and $k=0.01$. For uniform meshes the finite element approximation oscillates around the exact solution since the flow velocity $u$ is far larger than the conductivity $k$. Much of this undesirable behavior, however, may be avoided by appropriately

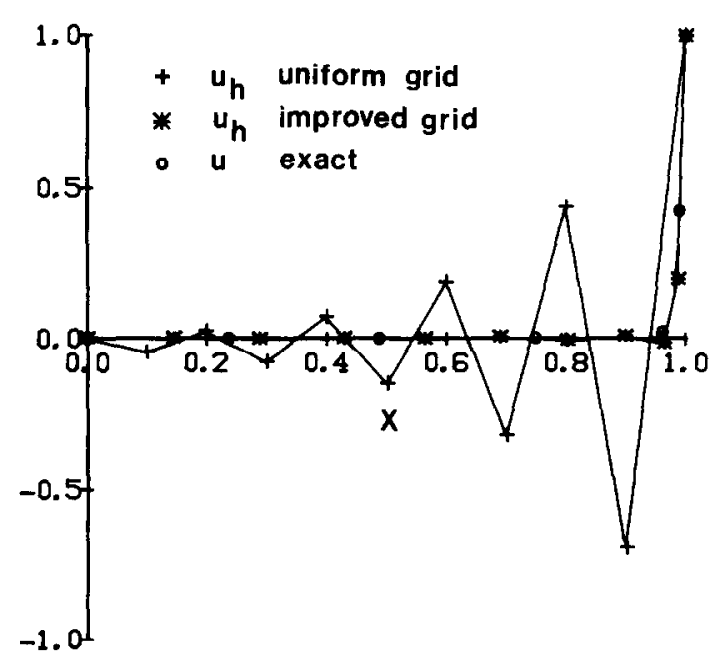

Fig. 1. Example 6.1. Finite element solutions $u_{h}$ corresponding to uniform and improved grids, and exact solution $u$.

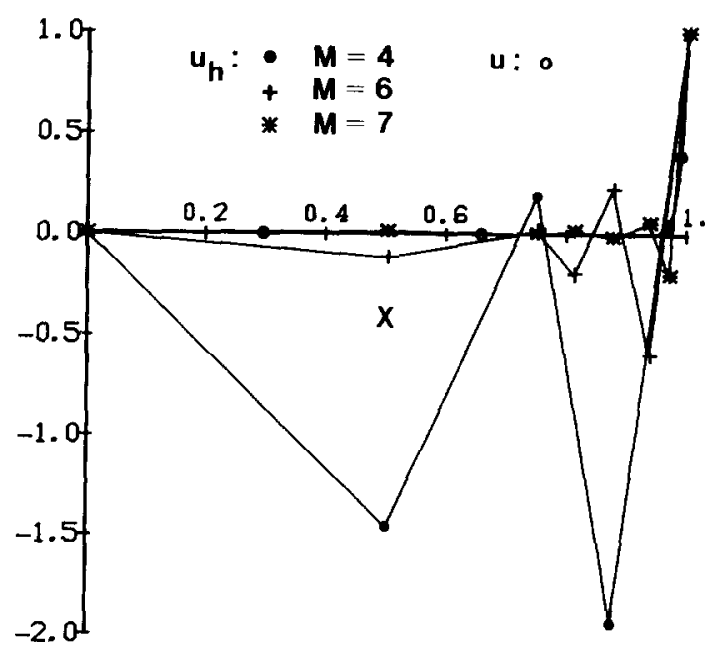

Fig. 2. Example 6.1. Finite element solutions for $h$-type of mesh rcfincment. $M=$ number of elements. 
modifying the grid. This observation is illustrated in Fig. 1 where the finite element solution corresponding to uniform and improved meshes are compared to the exact solution. The comparison shows that the oscillatory behavior of the approximation is substantially reduced by the improved grid. The finite element solution obtained from the improved grid is essentially undistinguishable from the exact solution.

In order to compare this result with other methods of grid improvement, an example of the application of an $h$-type method is shown in Fig. 2. Starting from a coarse grid, the mesh was enriched by subdividing elements in regions where the approximation was poorer. This Babuska's method also provides a substantial improvement of quality of approximate solutions.

One possible advantage of the method proposed in this article is that once the algorithm of mesh movement is established, it also works for time dependent problems. In this case, the finite element mesh moves as time $t$ goes. However, in the extension of the $h$-type method, it might be quite difficult to manage subdividing elements for time dependent problems when the 'shock' wave front travels as time goes.

EXAMPLE 6.2. For the second example the exact solution $u$ corresponds to the displacement inside a domain $\Omega$ under plane strain conditions. The domain $\Omega$ is given by

$$
\Omega=\left\{(x, y) \in \mathbb{R}^{2}: 0<x<2,0<y<1\right\}
$$

and boundary conditions are imposed as in Fig. 3. A Poisson's ratio $\nu=0.499$ is used, corresponding to an essentially incompressible material. The finite element approximation to the solution $u$ also exhibits an oscillatory behavior that can be improved using a graded mesh.

The oscillatory bchavior of the displacement near the fixed boundary is caused by the so called selective reduced integration method used to solve nearly incompressible problems. This method applies the 1-point Gaussian integration rule to evaluate the stiffness matrices related to the term of volumetric strain, while the $2 \times 2$ Gaussian integration rule is applied for the term of shear strain, for 4-node isoparametric quadrilateral elements.

A uniform mesh formed by bi-linear quadrilaterals (i.e. 4-node elements) was used as the initial grid and was modified using the guidelines given by (19) with $k=1$. In this case,

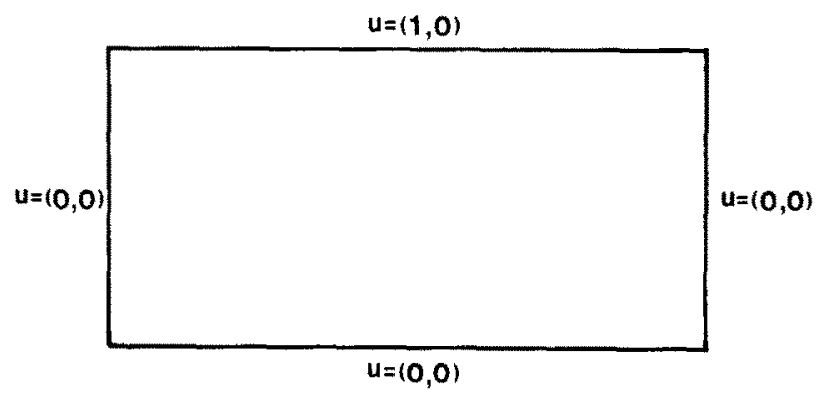

Fig. 3. Example 6.2. Boundary conditions. 
however, the exact solution $u$ is not smooth enough to be in $H^{2}(\Omega)$ and therefore, the conditions for the construction of the interpolation error bound (4) are not satisfied. The solution $u$ belongs to a larger space $H^{3 / 2}(\Omega)$ and a more conservative estimate of the bound is available by replacing $(k+1)$ by $\frac{3}{2}$ in (4) and in the definition of $\beta$ in the grid optimization guidelines (19). It has been observed, however, that the improved grids have the effect of isolating the singularities in $u$ and that the results obtained from the guidelines in (19) with $k+1=2$ or $k+1=\frac{3}{2}$ are essentially the same.

The oscillatory behavior of the approximation associated with the uniform mesh is shown in Fig. 4. Schemes such as selective reduced integration with filtering methods are normally used in an effort to improve this behavior. However, as an alternative, grid optimization can be used with success for this purpose, as evidenced in Fig. 5 which depicts the displacement field associated with the improved grid. The improved grid itself is shown in Fig. 6 . The initial value of $B^{2}(\hat{\boldsymbol{u}}, \hat{\boldsymbol{A}})\left(\frac{3}{2}\right.$ semi-norm $)$ is reduced by $30 \%$ after one iteration and by $40 \%$ after 5 iterations of the algorithm described earlier.

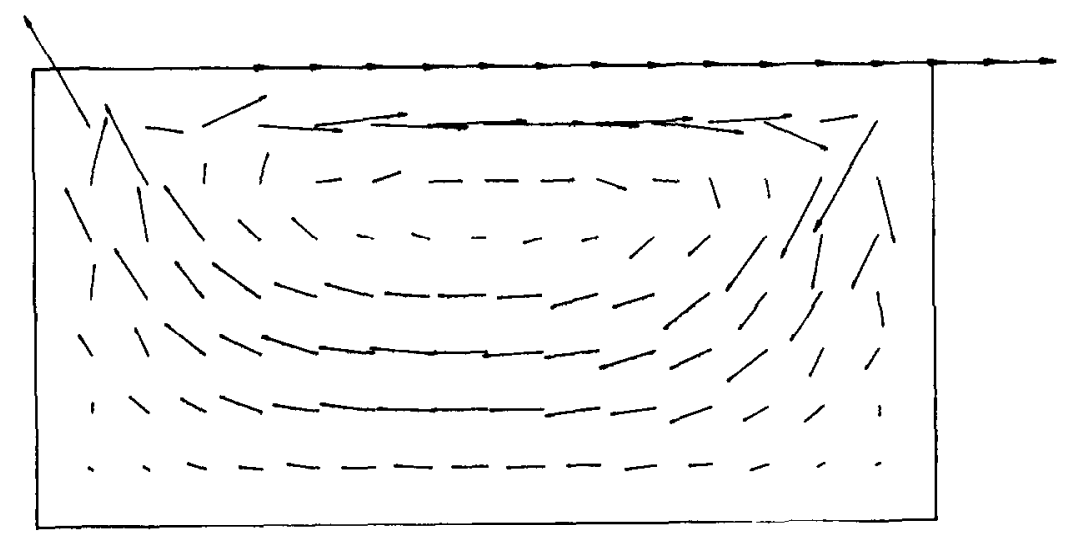

Fig. 4. Example 6.2. Displacement pattern associated with the uniform mesh.

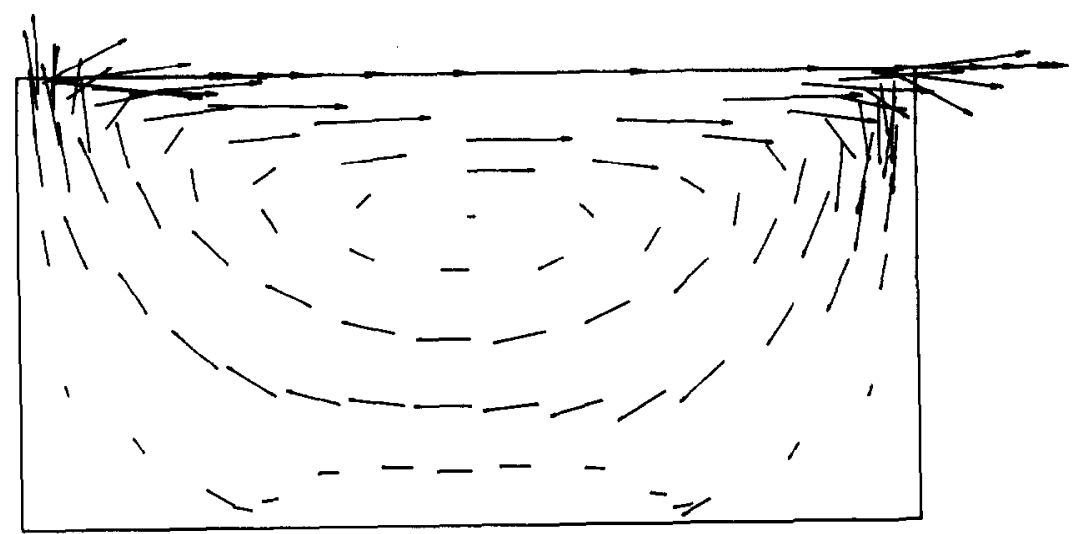

Fig. 5. Example 6.2. Displacement pattern associated with the improved grid. 


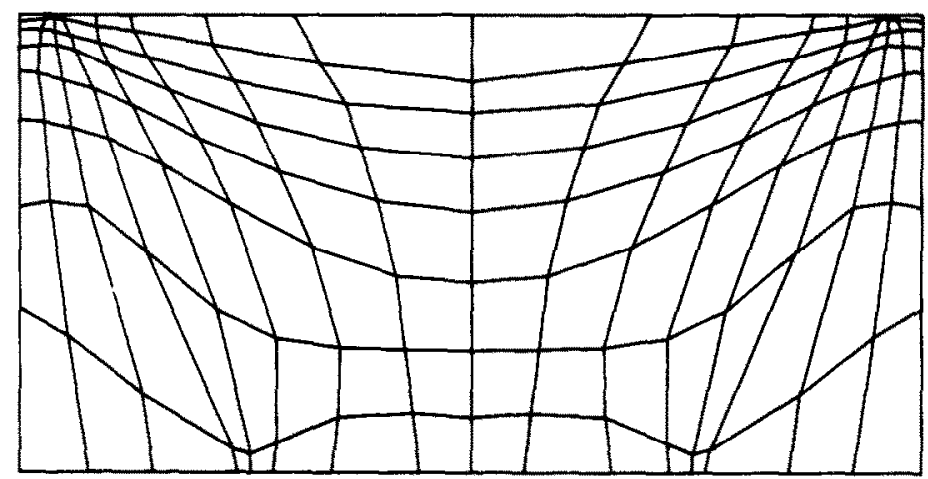

Fig. 6. Example 6.2. Improved grid.

EXAMPLE 6.3. The cracked plate under tension shown in Fig. 7 provides another example of the application of the grid optimization guidelines to problems with singularities. The finite element problem was solved using bi-linear, quadrilateral 4-node elements. The initial grid is shown in Fig. 8. The improved grid was obtained using the optimization guidelines (19) with $(k+1)=\frac{3}{2}$ to account for the singularity (Fig. 9). As in the previous example, essentially the same grid is produced by the usual choice of seminorms.

The changes performed on the initial grid have a substantial effect on the representation of the stresses near the crack. Fig. 10 shows the (normalized) magnitude of the equivalent stress (Tresca) as a function of the distance $r$ to the origin, for the ray $\theta=30^{\circ}$. The singularity at the

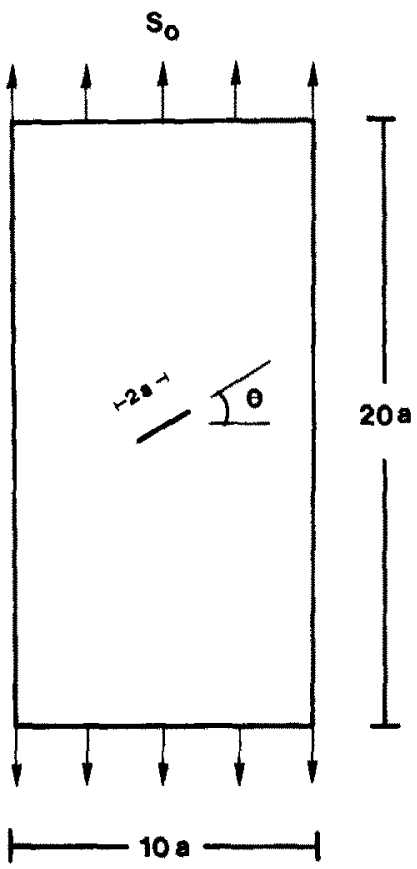

Fig. 7. Example 6.3. Plate geometry. $\theta=30^{\circ}$.

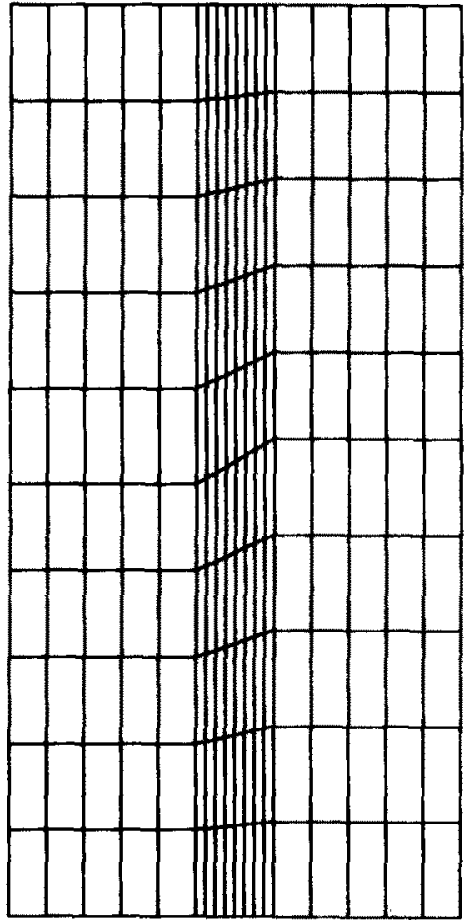

Fig. 8. Example 6.3. Initial grid. 


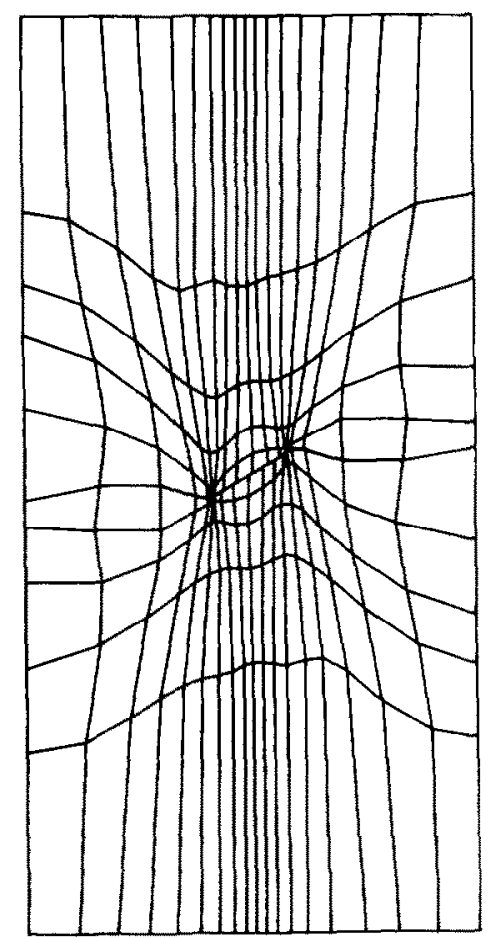

Fig. 9. Example 6.3. Improved grid.

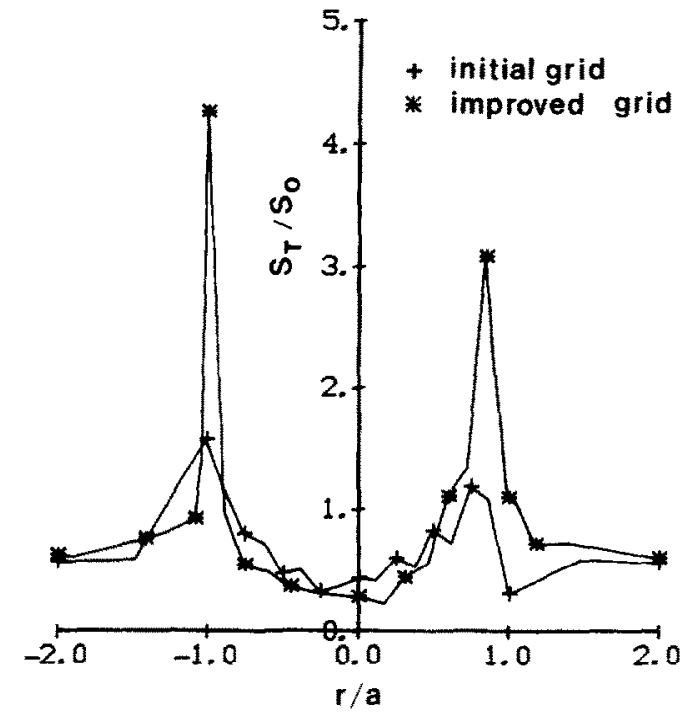

Fig. 10. Example 6.3. Stress distribution near the crack. Distance $r$ is measured from the center of the crack, on the ray $\theta=30^{\circ}$.

crack tip $(r / a= \pm 1)$ is better described by the improved grid, obtained after four iterations, which provides a maximum value of the ratio $S_{T} / S_{0} \approx 2.7$ times greater than the maximum available from the initial grid.

EXAMPLE 6.4. The last example in this article is for bending of a cantilevered beam under a tip load. To do this, element stiffness matrices are obtained by the following selective reduced integration scheme. Noting that the stress-strain relation for plane problems could be represented by two constants $\lambda$ and $\mu$ such that

$$
\left\{\begin{array}{c}
\sigma_{x} \\
\sigma_{y} \\
\sigma_{x y}
\end{array}\right\}=\left[\begin{array}{ccc}
\lambda & \lambda & 0 \\
\lambda & \lambda & 0 \\
0 & 0 & \mu
\end{array}\right]\left\{\begin{array}{c}
\varepsilon_{x} \\
\varepsilon_{y} \\
\gamma_{x y}
\end{array}\right\}+\left[\begin{array}{ccc}
2 \mu & 0 & 0 \\
0 & 2 \mu & 0 \\
0 & 0 & 0
\end{array}\right]\left\{\begin{array}{l}
\varepsilon_{x} \\
\varepsilon_{y} \\
\gamma_{x y}
\end{array}\right\},
$$

we compute the stiffness matrix related to the first part of the right-hand side of the above constitutive equation by using the 1-point Gaussian quadrature rule for 4-node bilinear quadrilateral elements, while we obtain the remaining stiffness matrix due to the second part by using the $2 \times 2$ (full) Gaussian integration rule. This selective reduced integration scheme is robust to both bending and incompressibility of materials. To be sensitive to the bending, the shear term $\sigma_{x y} \gamma_{x y}$ must be evaluated by the reduced integration scheme, and the term $\lambda \varepsilon_{k k} \varepsilon_{j j}$ related to the volumetric strain has to be reduced to avoid locking of approximate solutions.

Now suppose that the domain $\Omega$ is given by 


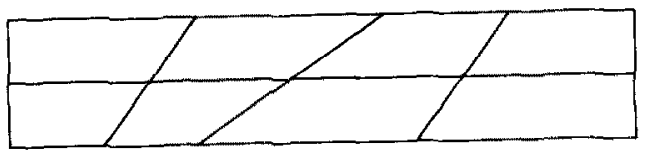

Fig. 11. Example 6.4. Initial grid.

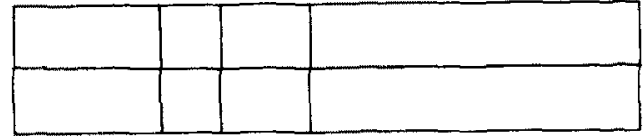

Fig. 12. Example 6.4. Improved grid.

$$
\Omega=\left\{(x, y) \in \mathbb{R}^{2}: 0<x<10,0<y<2\right\},
$$

and $\lambda=E \nu /\left(1-\nu^{2}\right), \mu=E / 2(1+\nu), E=10$ and $\nu=0.3$. If a point load $P=-1$ is applied at the top of the free end, elementary beam theory yields the deflection of the free end is $w=-P L^{3} / 3 E I, L=10$, and $I=\frac{1}{12} b h^{3}=\frac{2}{3}$, that is, $w=-50$. Using eight 4-node elements shown in Fig. 11, the deflection is computed as -30.6 at the free end. Applying grid optimizer described in above, we have the finite element model shown in Fig. 12 which provides the deflection -51.6 at the free end after three iterations.

\section{Conclusion}

The interpolation error associated with the finite element model constitutes a meaningful criterion for grid optimization. The analysis is simplified by the use of bounds on this error as the basis for the construction of objective functions and for the derivation of criteria for optimality. Although some approximations are necessary in order to render these criteria computationally useful, the resulting expressions remains intimately related to the original measure of interpolation error. The guidelines for grid optimization suggested here appear in a form that is attractive for computations and can lead to good improvements in the quality of the finite element solution with relatively small effort.

\section{Appendix A}

The stationary conditions (17) for problem (10) are derived after performing a first-order variation of the objective function $B^{2}$ with respect to element areas. The procedure is outlined here for two-dimensional grids but it can be followed also to derive the one-dimensional result.

For convenience, let the positive quantity $I_{K}$ and function $g(t)$ be defined by

$$
I_{K}=\int_{K} g(u(t)) \mathrm{d} t \equiv|u|_{k+1, K}^{2} .
$$

Let $\boldsymbol{A}=\left(A_{1}, A_{2}, \ldots, A_{M}\right)$ be a feasible vector of element areas and let $\delta \boldsymbol{A}=$ $\left(\delta A_{1}, \delta A_{2}, \ldots, \delta A_{M}\right)$ represent a small variation consistent with the constraints (10.b) and (10.c), that is,

$$
\sum_{K}\left(A_{K}+\delta A_{K}\right)=1
$$


with $A_{K}>0$ for all $K=1,2, \ldots, M$. Let $\delta A_{K N}$ be the variation of the area $A_{K}$ with respect to changes in $A_{N}$. With this notation,

$$
\delta A_{K}=\sum_{N=1}^{M} \delta A_{K N}
$$

and $\delta A_{K N}=-\delta A_{N K}$, with the conventions

$$
\delta A_{K K}=0
$$

and $\delta A_{K N}=0$ if $K \cap N=\emptyset$.

If the solution $u$ is smooth enough, there exist positive quantities $g_{K N}$ such that the change produced in $I_{K}$ by the variation $\delta A$ is, to first order, given by

$$
\delta I_{K}=\sum_{N-1}^{M} \delta I_{K N} \equiv \sum_{N=1}^{M} g_{K N} \delta A_{K N}, \quad K=1,2, \ldots, M
$$

The magnitude of $g_{K N}$ is related to the values of $g(u(t))$, on the interface between elements $K$ and $N$, that is, to the mean square value of the $(k+1)$ derivatives of $u$. For instance, let $K$ and $N$ share two (primary) nodes $a$ and $b$ and, let $\delta a$ and $\delta b$ be the motion of the nodes associated with $\delta \boldsymbol{A}$. Then

$$
\delta A_{K N}=\frac{1}{2}\|b-a\|(\delta a+\delta b) \cdot n, \quad \delta I_{K N}=\frac{1}{2} \int_{a}^{b} g(u) \mathrm{d} s[(\delta a+\delta b) \cdot n] .
$$

From which

$$
g_{K N}=\frac{\int_{a}^{b} g(u) \mathrm{d} s}{\|b-a\|} .
$$

The unit vector $n$ is normal to the side $a b$ in the outward direction to $K$. Similarly, if $K$ and $N$ share only one node $b$, the magnitude of $g_{K N}$ is given simply by $g(u(b)$ ).

With the notation described above, the first variation of the objective function is obtained after expanding

$$
B^{2}(u, \boldsymbol{A}+\delta \boldsymbol{A})=\sum_{K=1}^{M}\left(A_{K}+\sum_{N=1}^{M} \delta A_{K N}\right)^{\beta}\left(I_{K}+\sum_{N=1}^{M} g_{K N} \delta A_{K N}\right) .
$$

From which

$$
\delta B^{2}=\sum_{K=1}^{M} \sum_{N=1}^{M}\left(A_{K}^{\beta} g_{K N}+\beta A_{K}^{\beta-1} I_{K}\right) \delta A_{K N} .
$$

Setting $\delta B^{2}=0$ for arbitrary but feasible $\delta A_{K N}$ 's implies that

$$
A_{K}^{\beta} g_{K N}+\beta A_{K}^{\beta-1}|u|_{k+1, K}^{2}=A_{N}^{\beta} g_{K N}+\beta A_{N}^{\beta-1}|u|_{k+1, N}^{2}
$$

for all $K$ and all $N$ such that $K \cap N \neq \emptyset$. 


\section{References}

[1] I. Babuska and W.C. Rheinboldt, A posteriori error estimates for the finite element method, Internat. J. Numer. Meths. Engrg. 12 (1978) 1597-1615.

[2] I. Babuska and W.C. Rheinboldt, Analysis of optimal finite-element meshes in R1. Math. Comput. 33 (1979) $435-463$.

[3] I. Babuska and W.C. Rheinboldt, Reliable error estimation and mesh adaptation for the finite element method, in: J.T. Oden, ed., Computational Methods in Nonlinear Mechanics (North-Holland, Amsterdam, 1980) 67-108.

[4] I. Babuska and B. Szabo, On the rates of convergence of the finite element method, Internat. J. Numer. Meths. Engrg. 18 (1982) 323-341.

[5] G. Sewell, An adaptive computer program for the solution of $\operatorname{DIV}(P(X, Y) G R A D U)=F(X, Y, U)$ on a polygonal region, in: J.R. Whiteman, ed., The Mathematics of Finite Elements and Applications II, MAFELAP 1975 (Academic Press, London, 1976).

[6] O.C. Zienkiewicz, D.W. Kelly, J.P. de S.R. Gago and I. Babuska, Hierarchical finite element approaches, error estimates and adaptive refinement, Proc. MAFELAP 1981, Brunel University, Uxbridge, U.K.

[7] O.C. Zienkiewicz, J.P. de S.R. Gago and D.W. Kelly, The hierarchical concept in finite element analysis, Comput. \& Structures 16 (1983) 53-65.

[8] A.G. Peano, Hierarchies of conforming finite elements for plane elasticity and plate bending, Comput. Math. Appl. 2 (1976) 221-224.

[9] B.A. Szabo and A.K. Mehta, p-convergent finite element approximations in fracture mechanics, Internat. J. Numer. Meths. Engrg. 12 (1978) 551-560.

[10] E.R. de Arantes e Oliveira, Optimization of finite element solutions, Proc. 3rd. Conf. Matrix Methods in Structural Mechanics, Wright-Patterson Air Force Base, OH, 1971.

[11] D.J. Turcke and G.M. McNeice, Guidelines for selecting finite element grids based on an optimization study, Comput. \& Structures 4 (1974) 499-519.

[12] D.J. Turcke, Characteristics of piecewise approximation in numerical analysis, in: M.S. Shephard and R.H. Gallagher, eds., Finite Element Grid Optimization ASME Special Publication PVP-38 (ASME, New York, 1979) 15-26.

[13] C.A. Felippa, Optimization of finite element grids by direct energy search, Appl. Math. Modelling 1 (1976) 93-96.

[14] C.A. Felippa, Numerical experiments in finite element grid optimization by direct energy search, Appl. Math. Modelling 1 (1977) 239-244.

[15] R.J. Melosh and P.V. Marcal, An energy basis for mesh refinement of structural continua, Internat. J. Numer. Meths. Engrg. 11 (1977) 1083-1091.

[16] M.S. Shephard, R.H. Gallagher and J.F. Abel, The synthesis of near-optimum finite element meshes with iteractive computer graphics, Internat. J. Numer. Meths. Engrg. 15 (1980) 1021-1039.

[17] M.S. Shephard, Finite element grid optimization--a review, in: M.S. Shephard and R.H. Gallagher, eds., Finite Element Grid Optimization ASME Special Publication PVP-38 (ASME, New York, 1979) 1-14.

[18] P.G. Ciarlet, The Finite Element Method for Elliptic Problems (North-Holland, Amsterdam, 1978). 